\title{
Spinal Cord Degeneration Associated with Type II Decompression Sickness: Case Report
}

I. M. Calder, MD ${ }^{1}$, A. C. Palmer, MA, ScD, FRCVS ${ }^{2}$, J. T. Hughes, MD, FRCP, FRCPath ${ }^{3}$, J. F. Bolt, MB, BS, DA ${ }^{4}$, J. D. Buchanan, MA, MB, BChir, DCP, MRCPath, RN ${ }^{5}$

${ }^{1}$ Institute of Pathology, London Hospital Medical College, London, UK. ${ }^{2}$ Department of Clinical Veterinary Medicine, Madingley Road, Cambridge, UK. ${ }^{3}$ Department of Neuropathology, Radcliffe Infirmary, Oxford, UK. ${ }^{4}$ Cobo and St Martin's Medical Practice, Clos de Carteret, Cobo, Guernsey, CI. ${ }^{5}$ Department of Pathology, Royal Naval Hospital Haslar, Gosport, Hants PO12 2 AA, UK.

\section{Summary}

The medical history, clinical and neuropathological findings at necropsy are described in a 50-year-old male amateur diver who suffered from Type II decompression sickness, a spinal 'bend'. He survived as a paraplegic for 4 years.

In the spinal cord upward Wallerian degeneration in the posterior columns and downward degeneration in the corticospinal tracts was explained by multiple small and medium sized infarcts affecting the centripetal blood supply to the cord. There was preservation of a rim of subpial fibres on the surface of the posterior and lateral columns. The grey matter and nearby white matter (supplied by the centrifugal arterial supply) was unaffected.

Key words: Decompression sickness; Spinal cord degeneration.

Decompression sickness (the 'bends') is arbitrarily divided into two types on the basis of the severity of clinical signs. Type I describes temporary joint pain, pruritus and abnormalities of cutaneous circulation, signs which usually improve after recompression. The manifestations of Type II DCS are more serious and include neurological deficits (spinal paralysis), respiratory involvement (the 'chokes') and hypovolaemic shock.

Although Type II decompression sickness (DCS) is known to cause spinal paralysis, there have been only four detailed reports of the long term pathological changes. The first two of these described predominant lesions in the posterior and lateral white columns but the grey matter was relatively unaffected (Van Rensselaer, 1891; Sharples, 1894). The root entry zone was seen to be damaged in the case described by Sharples. The third report (Lichtenstein and Zeitlin, 1936) concerned a caisson worker who died 25 years after spinal paraplegia from which he had partially recovered; he was able to walk with the aid of a stick but was impotent. Pathologically both the posterior and lateral columns were affected with some preservation of the subpial myelin. The pattern of tract degeneration was ascribed by the authors to a primary destructive lesion in the cord in the middle thoracic region. 
In 1981 Palmer et al. described the pathology in the spinal cord of an amateur diver who died 4 years after a decompressive episode. Although initially partially paralysed the patient regained almost normal motor function. He was virtually without symptoms but on examination there were slight upper motor signs affecting the face and both upper and lower limbs. Long tract degeneration was found in the spinal cord affecting the posterior and lateral colums from $\mathrm{C} 1$ to L4, believed to arise from a primary site of damage between C7 and T4.

We now wish to report the pathological appearance in the spinal cord of an amateur diver who survived for 4 years after a major decompressive diving accident.

\section{Case Report}

The patient was a 50-year-old man who had been diving over a period of 2 years; he was self-taught. On the day prior to the accident he had dived on two occasions to a depth of $120^{\prime}$ - he was using a depth meter. On the day of the accident he was said to have dived first to $120^{\prime}$ and 40 minutes later he dived for a second time to a depth of $130^{\prime}$, with a total decompression time (on the first dive) of 4 minutes. In the history there is a suggestion that both dives on the day may have been to a depth of $145^{\prime}$. Several days later the patient recalled that on his last dive he had 'blacked out' as he came up to a depth of $20^{\prime}$; his son confirmed that he had surfaced quicker than normal over the last $20^{\prime}$. Three minutes after surfacing he suffered chest pain, double vision, vertigo, nausea, vomiting, clumsiness and a loss of memory. He became comatose. He was admitted to the Decompression Unit of the St John's Ambulance and Rescue Service, Guernsey, at 12:20 (day 1). On examination he was amnesic and barely accessible; he had gross nystagmus and was clumsy. He complained of chest pain, nausea and vomiting. He was cold, pale and in peripheral circulatory failure.

The patient was recompressed and at a pressure corresponding to $30^{\prime}$ of sea water (fsw) his memory suddenly cleared and he became coherent. When examined at 60 fsw some 20 minutes after admission his chest and heart sounds were normal, pulse rate was 90 per minute and of small volume and jugular venous pressure was $0 \mathrm{mmHg}$. On neurological examination he was found to be using his left hand. His pupils were equal and reacted to light, there was spontaneous nystagmus in both directions and hearing appeared normal. There was no facial weakness and both tongue and palate were central. Limb tone was increased and muscle power decreased but this was ascribed to lack of mental concentration. Hand tapping and heel-shin test were clumsy. He was noticed then to prefer to use his right hand. Deep reflexes in both upper and lower limbs were increased, especially the knee jerk. Plantar reflexes were downward. The upper abdominal reflexes were absent. Conscious reaction to pinprick and to vibration were normal.

A diagnosis was made of Type II decompression sickness affecting the vestibular apparatus, the cerebrum and the spinal cord, complicated by a small cerebral air embolism.

The patient was given intravenous fluids; 1.01 Hartmann's solution, 0.51 of dextran 40 and Heparin 2500 i.u. Dexamethasone $12.5 \mathrm{mg} \times 2$ plus $0.5 \mathrm{mg}$ orally. He was decompressed on Table 62 with 30 minutes of oxygen repeated for 1 hour-a total of $1 \frac{1}{2}$ hours on oxygen with 5 minute intervals every 25 minutes.

During the afternoon he showed steady improvement and by 2 hours 10 minutes after admission he had passed 21 of urine. When decompression was at $5 \mathrm{fsw}$ equivalent he was able to stand, was clear-headed but his leg movement was clumsy. He was re-examined 6 hours 40 minutes after admission. His reflex activity was unchanged in the upper limbs, but he showed increased ankle jerks and upgoing plantars. However he was sweating profusely. To combat probable spinal cord oedema he was given further Dexamethasone intravenously. Heparin 2500 i.u. was also given to reduce the risk of free fatty acids increasing platelet stickiness. He was recompressed on Table RN 54 to 165 fsw.

Next morning (day 2) still in the chamber at $60 \mathrm{fsw}$ he felt better but had stiff ataxic lower limbs. The left plantar was upgoing and the right equivocal; there was marked ankle clonus on both sides. The upper extremities appeared normal with good grip and co-ordination. Urine output was good. The intravenous therapy was continued and by evening the patient had reached a steady state with spastic lower limbs but downgoing plantar reflexes. By 08:30 the following morning (day 3) the decompression had reached $30 \mathrm{fsw}$. He was given oxygen by mask for 25 minutes with 5 minute breaks. He was due to be taken out of the chamber by noon. By then he had weak volitional movements in the flexors and extensors of the hips and he could move all his toes. There was loss of proprioception, vibration sense and pinprick symmetrically below the level supplied by T12. 
By the third day after the onset of Type II DCS the upper extremities appeared normal. There was variable loss of pinprick from the lower abdominal wall and loss of position and possible vibration sense from the right leg whereas these modalities were normal in the left leg.

Over the next 18 days the patient was given daily hyperbaric oxygen treatment for 1 hour at a pressure of $30 \mathrm{fsw}$. For the subsequent 3 weeks this treatment was given every other day.

By the fourth day after the decompression episode, spasms occurred in his legs; pinprick was present as far down as the knees and vibration sense was present at the level of the tibial tuberosities. Power in the quadriceps and hamstrings gradually improved although by the fifth day there was still minimal power in the dorsiflexors of the foot. The right knee jerk was normal, the left increased and the abdominal reflex was present in the lower left quadrant. Position sense was $100^{\circ}{ }_{0}$ normal but the right hand was still clumsy. Function of the cranial nerves was normal.

The patient continued to improve. His muscular activity was noticeably better under hyperbaric conditions where he was able to move his lower limbs. One week after the diving accident he had activity in the quadriceps and hamstrings, a little power in the calves but dorsiflexion continued poor. Deep reflexes were brisk but described as non-pathological although there was unsustained clonus of the right ankle. The abdominal reflex was absent from the right quadrant and the plantar reflexes were again upgoing.

Over the subsequent 6 days there was gradual improvement, vibration sense being better on the left side than on the right.

Twenty four days after the accident the lower limbs were less spastic and there was partial flexion at the knees. On both sides dorsiflexion was weak but plantar flexion quite powerful. Deep reflexes of the upper and lower limbs had again increased (especially the biceps reflex), position sense was normal but pinprick variable. The right plantar was equivocal, the left was upwards.

Two weeks later he began to walk between bars. The left leg was more spastic than the right but the patient was aware of light and deep touch. Thereafter he suffered from retention of urine with overflow and had to be catheterised on a number of occasions.

Eleven months after the original insult he was readmitted because of infection in the urinary tract which was probably exacerbated by the presence, adhered to the trigone, of a soft calculus which was crushed and washed out.

During the ensuing years he made some progress and was able to walk slowly with the aid of a stick. He died 4 years after the diving accident from coronary thrombosis.

\section{Necropsy}

External: Adequately nourished caucasion male, $180 \mathrm{~cm}$ tall with slight wasting of muscles of lower limbs.

Cardiovascular: Heart weighted $360 \mathrm{~g}$ with areas of old infarction of anterior wall of left ventricle. Severe atheroma throughout coronary arteries with left coronary artery occluded by thrombus just distal to the origin.

Lungs: Congestion. No pulmonary embolus.

The cause of death was given as acute myocardial ischaemia due to coronary artery thrombosis consequent on atheroma.

Central nervous system: Brain weighed $1430 \mathrm{~g}$ with congestion of meninges.

\section{Pathological findings in the spinal cord}

\section{Methods}

The spinal cord was removed from the vertebral canal at necropsy and fixed in $10^{\circ}{ }_{0}$ formol saline. Transverse tissue blocks for paraffin embedding were taken from representative levels.

Paraffin sections were stained with a simple tissue stain (haematoxylin and eosin) for myelin (Methasol fast blue) and where necessary for axons and cell bodies (Holmes silver stain) and glia (Holtzer and PTAH). In addition Marchi preparations were made on the following segmental levels: C3, T5, T11, L1 and L3. 


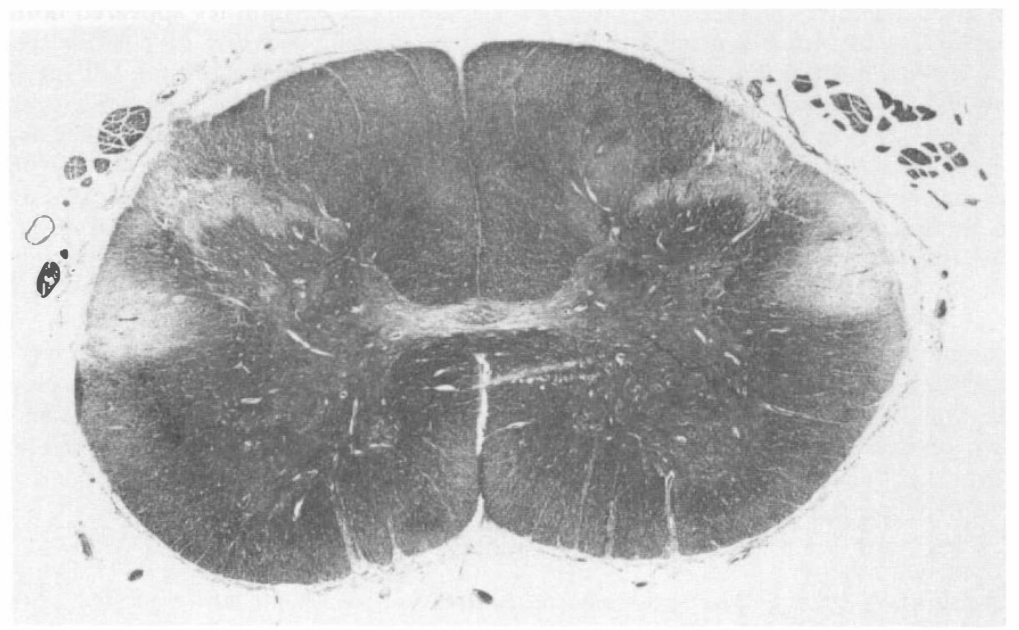

Figure 1 Cervical cord (C7) showing degeneration in the lateral corticospinal tracts. Methasol fast blue $\times 14$.

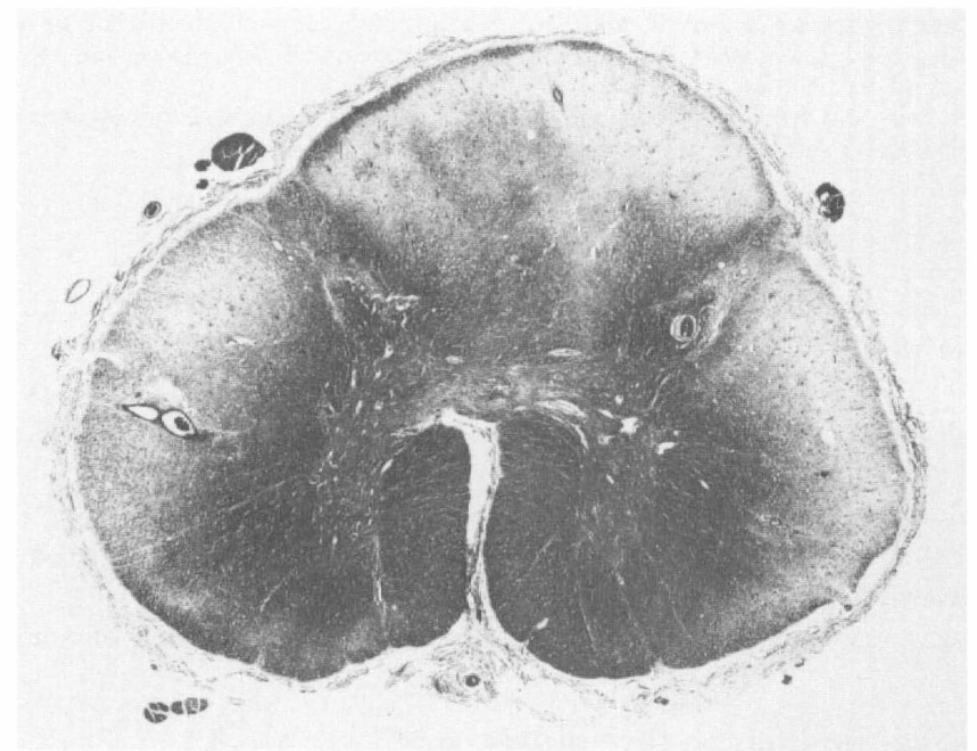

Figure 2 Cervical cord (C3) there is degeneration in the posterior and lateral columns. Note the preservation of the subpial white matter. Methasol fast blue $\times 14$.

\section{Results}

The sections showed widespread and profound damage to the spinal cord. In the long tracts this was seen as downward degeneration in the corticospinal tracts and upward degeneration in the posterior columns (Fig. 1, Fig. 2). The nature and pattern of this long tract degeneration indicated that it was caused by numerous widespread lesions in the cord with a contribution from damage to 
the posterior nerve roots. The posterior column degeneration was almost complete in the cervical region. However there was some sparing of the central fibres of the fasciculus gracilis from the lowest sacral segments and an interesting peripheral rim of unaffected fibres (Fig. 2) in the posterior columns and in the lateral columns. This upward Wallerian degeneration in the posterior columns arose from numerous small and medium size irregular lesions which were often perivascular in distribution. They had the appearance of old infarcts arising in the territories of the short and long penetrating vessels proceeding inwards radially into the posterior columns. Some of the posterior roots also showed loss of nerve fibres with hyalination of small vessels accompanying these roots. The corticospinal tract degeneration began in the cervical region and increased to a maximum of almost total degeneration of all the fibres in the lumbar cord and below. This was also related to small and medium size old infarcts surrounding small hyalinised vessels.

The grey matter of the spinal cord was largely spared by the pathological process and the anterior spinal artery, vein and their branches and tributaries were patent and normal. The subarachnoid space was obliterated by an acellular fibrous reaction indicating old damage now quiescent.

This pattern of spinal cord damage affecting predominantly the white matter and with small and medium size infarcts around hyaline vessels, is that of a vascular disease affecting the centripetal blood supply of the spinal cord in which the short and long penetrating vessels are focally occluded. The interesting preservation of a rim of fibres of the posterior and lateral columns is likely to have a vascular explanation and has been reported in experimented DCS Type II in the goat (Palmer et al., 1978). The preservation of the grey matter and the intact patent state of the anterior spinal artery and its branches suggest that this component of the vascular supply (the centrifugal) was spared.

The detailed histology of the direct lesions and those of the long tract degeneration is that of a now quiescent picture mainly of fibrous gliosis with a few lipid phagocytes.

\section{Results by the Marchi method}

The blocks and frozen sections examined by Marchi were valuable in confirming the position of the tract degenerations. The findings were less evident than in the myelin stains because of the long interval between the cord damage and which had allowed quantities of Marchi positive material to be removed by macrophages now seen around blood vessels (Fig. 3). Marchi positive material at the root entry zone was conspicuous by this technique and confirmed the additional component of damage to the tract of Lissauer in this case.

\section{Discussion}

The clinical signs shown by the patient can be related to the profound damage in the spinal cord affecting principally the posterior and lateral columns, a pattern of injury similar to that of previous reports of DCS. The pathogenesis of the lesions in our case is ascribed to multiple small and medium sized infarcts within these tracts. It is noteworthy that the grey matter was unaffected and this suggests that the centrifugal blood supply was unaffected. 


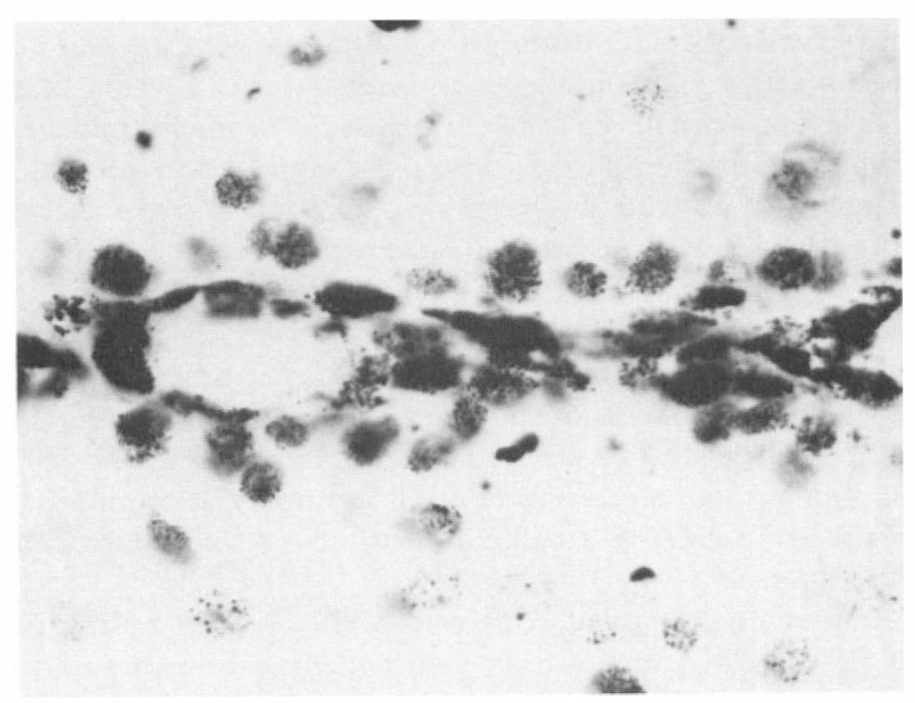

Figure 3 Thoracic cord, lateral columns. Marchi-stained material within phagocytes arranged around a vessel. Marchi $\times 300$.

A similar pattern of tract degeneration follows experimental DCS in the goat (Palmer et al., 1976), the dorsal columns being especially affected. Involvement of the lateral columns is not usually so extensive in this species because the lateral corticospinal tract only reaches lower cervical levels.

As with DCS in man experimental DCS in the goat leads to severe malacia of the white matter of the cord with a relative preservation of an outer rim of myelin. In the goat there is good evidence that the spinal cord infarcts stem from microthrombus formation associated with platelet aggregation and vasogenic oedema, which presumably arise from intravascular gas bubble formation (Palmer et al., 1978). The preservation of the subpial myelin probably reflects an intact meningeal arterial supply.

The infarcts in man probably arise in a similar fashion. An absence of an adequate collateral circulation in the white matter of the spinal cord is likely to make the myelin especially vulnerable to this type of lesion.

\section{Acknowledgements}

This work was carried out with the help of a grant from the Medical Research Council to one of us (ACP). We wish to thank Mrs I. S. Lloyd, Miss P. Deacon and Mr J. E. Payne for technical assistance and also Mrs E. Williams for help with the typescript.

\section{References}

Lichtenstein BW, Zeitlin H 1936 Caisson disease a histologic study of late lesions. Archives of Pathology 22:86-98.

Palmer AC, Blakemore WF, Greenwood AG 1976 Neuropathology of experimental decompression (dysbarism) in the goat. Neuropathology and Applied Neurobiology 2:145-156.

Palmer AC, Blakemore WF, Payne JE, Sillence A 1978 Decompression sickness in the goat: nature of brain and spinal cord lesions at 48 hours. Undersea Biomedical Research 5:275-286. 
Palmer AC, Calder IM, McCallum Ri, Mastaglia FL 1981 Spinal cord degeneration in a case of 'recovered' spinal decompression sickness. British Medical fournal 283:888.

SHARPLES CW 1894 A contribution to the pathology of the spinal cord in diver's palsy. Fournal of Nervous Diseases 19:636-640.

VAN RENSSELAER H 1891 The pathology of the caisson disease. Medical Record 40:141-182. 